\title{
Towards Characterization of Wireless Traffic in Coexisting 802.11a/g and 802.11n Network
}

\author{
Hirochika Asai \\ University of Tokyo
}

\author{
Kensuke Fukuda \\ NII / PRESTO JST
}

\author{
Hiroshi Esaki \\ University of Tokyo
}

\section{INTRODUCTION}

The number of wireless devices and the traffic volume generated by these devices become significant today, and many devices begin supporting 802.11n protocol for higher-speed wireless access. However, the diversification in link types of end-hosts may degrade system performance. For example, hosts using 802.11 protocols had better not be relay nodes in a P2P live streaming system because 802.11 is a half-duplex protocol and usually less stable compared to modern wired links. Hence, understanding traffic characteristics of various link types is essential for improving or building network architectures. Moreover, estimation of the link type of a remote host possibly achieves better performance (e.g., higher throughput) in some network systems. Baiamonte et al. [1] have proposed an algorithm to detect wireless hosts from passive measurement by using the entropy of packet interarrival time (PIT). Wei et al. [2] have also proposed an algorithm to classify access network types. However, these algorithms pay no attention to new link types such as 802.11n, 3G, and WiMAX even though each of them has different characteristics and possibly degrades the performance of network systems.

Our goal is to analyze and model the characteristics of various link types which can be criteria for system design, and then to provide an algorithm to identify the link type of a remote host. In this paper, we analyze PIT, its entropy of 802.11 hosts, and fairness between $802.11 \mathrm{a} / \mathrm{g}$ and $802.11 \mathrm{n}$ in coexisting these wireless networks with packet trace in relatively large-scale experiment. The contributions of this paper are to show that $802.11 \mathrm{n}$ has different characteristics from $802.11 \mathrm{a} / \mathrm{g}$ in

Permission to make digital or hard copies of all or part of this work for personal or classroom use is granted without fee provided that copies are not made or distributed for profit or commercial advantage and that copies bear this notice and the full citation on the first page. To copy otherwise, to republish, to post on servers or to redistribute to lists, requires prior specific permission and/or a fee.

ACM CoNEXT Student Workshop, November 30, Philadelphia, USA.

Copyright 2010 ACM 978-1-4503-0468-9/10/11 ...\$10.00.
PIT and its entropy, and to confirm fairness between $802.11 \mathrm{a} / \mathrm{g}$ and $802.11 \mathrm{n}$ hosts in time-domain.

\section{TRAFFIC CHARACTERISTICS}

The entropy of PIT has been commonly used to characterize the traffic of bottleneck links $[1,2]$. A probability mass function (PMF) of PIT is defined as $P\left(\tau_{i}\right)=$ $m_{i} / m$, where $m$ is the total number of sampled PIT and $m_{i}$ is the number of samples whose PIT is in the range $\left[\tau_{i}, \tau_{i+1}\right)$. N.B., $\tau_{i}=b i$, where the time bin $b$ is a constant value. We then define the entropy of PIT from the PMF in the equation: $H:=-\sum_{i} P\left(\tau_{i}\right) \log _{2} P\left(\tau_{i}\right)$. The entropy in this context represents uncertainty of PIT; for example, PIT of hosts connecting with shared links might be fluctuated due to collisions and the entropy would be larger while those connecting with nonshared (i.e., exclusive) and stable links can certainly send packets without collisions nor loss. In this paper, we use the same parameters as those used in Ref. [1], that is, the time bin $b$ is $100 \mu s$, a maximum threshold of PIT is set to $10 \mathrm{~ms}$, a time window for calculation of entropy is $20 s$, and a minimum threshold of the number of samples in a time window is 200 .

We also define two fairness indexes, throughput and transfer duration fairness indexes, to evaluate fairness between $802.11 \mathrm{a} / \mathrm{g}$ and $802.11 \mathrm{n}$ hosts in coexisting these networks. The throughput fairness index of the protocol $p(p \in\{802.11 \mathrm{a} / \mathrm{g}, 802.11 \mathrm{n}\})$ with the channel $c$ is defined as $F_{s}^{p, c}(\Delta):=\frac{s_{c}^{p}(\Delta)}{s_{c}^{802.11 \mathrm{a} / \mathrm{g}}(\Delta)+s_{c}^{802.11 \mathrm{n}}(\Delta)}$, where $s_{c}^{p}(\Delta)$ is average throughput among hosts using the protocol $p$ with the channel $c$ during the duration $\Delta$. In the same way, the transfer duration fairness index is also defined as $F_{d}^{p, c}(\Delta):=\frac{d_{c}^{p}(\Delta)}{d_{c}^{802.11 \mathrm{a} / \mathrm{g}}(\Delta)+d_{c}^{802.11 \mathrm{n}}(\Delta)}$, where $d_{c}^{p}(\Delta)$ is average transfer duration among hosts using the protocol $p$ with the channel $c$ during the duration $\Delta$.

\section{MEASUREMENT AND RESULTS}

The measurement was conducted in the biannual symposium of the WIDE project in 9-12 March 2010; the wireless network of the symposium consisted of nine wireless access points (APs: Cisco Aironet 1250) with 


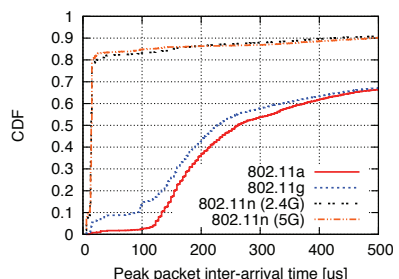

(a) Peak PIT

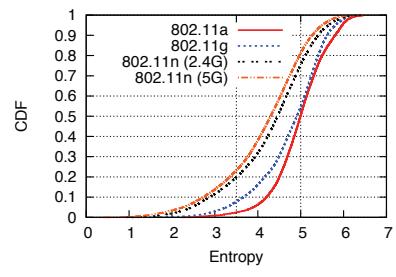

(b) Entropy
Figure 1: CDF of peak PIT and entropy of PIT by protocols

one controller (Cisco Wireless Controller 5508), and 215 (client) stations. All the APs were operated in lightweight mode, and consequently, all the data frames through APs were encapsulated by the CAPWAP protocol (RFC 5415) and went through the controller. We had captured these encapsulated data frames at a monitored interface between the controller and APs by tcpdump (total: 122 million frames). After the measurement, we extracted 802.11 frames from the encapsulated traffic trace. We had also measured the information of associated stations from APs every ten seconds by SNMP. The maximum number of stations simultaneously connected to APs was 148 . The number of measured associations of $802.11 \mathrm{a}, 802.11 \mathrm{~g}, 802.11 \mathrm{n}(2.4 \mathrm{GHz})$ and $802.11 \mathrm{n}(5 \mathrm{GHz})$ are $105,129,129$ and 116, respectively; N.B., we double-counted the hosts that support both $2.4 \mathrm{GHz}$ and $5 \mathrm{GHz}$ etc.

We show the cumulative distribution function (CDF) of peak PIT by protocols in Figure 1(a). The peak PIT is the peak value in the PMF $P$ with the time bin $b=1$. We confirmed that the peak PIT of $802.11 \mathrm{a} / \mathrm{g}$ mostly distributed above $120 \mu \mathrm{s}$, and that of $802.11 \mathrm{n}$ concentrated around $10 \mu s$, meaning that the block ACK mechanism in $802.11 \mathrm{n}$ affected PIT significantly. We then show the CDF of the entropy of PIT by protocols in Figure 1(b). The entropy of $802.11 \mathrm{n}$ has smaller values than that of $802.11 \mathrm{a} / \mathrm{g}$. The result points out that the identification algorithm in Ref. [1] makes inaccurate annotations to more than $20 \%$ hosts though it is appropriate for $802.11 \mathrm{a} / \mathrm{g}$ because it judges a host with $H \leq 3.5$ as "wired". Thus, the simple entropy-based algorithm has a difficulty in identifying $802.11 \mathrm{n}$ hosts.

We also evaluate fairness between $802.11 \mathrm{a} / \mathrm{g}$ and $802.11 \mathrm{n}$ hosts in coexisting these networks. 802.11a and 802.11n share the $5 \mathrm{GHz}$ band, and $802.11 \mathrm{~g}$ and $802.11 \mathrm{n}$ also share the $2.4 \mathrm{GHz}$ band, implying the potential conflicts (i.e., fairness issue). In this evaluation, we focus on the channel 36 in $5 \mathrm{GHz}$ band. Figures $2(\mathrm{a})$ and (b) show the probability distribution functions (PDF) of throughput and transfer duration fairness indexes for each second by protocols, respectively. Here, throughput is calculated from the accumulated frame length,

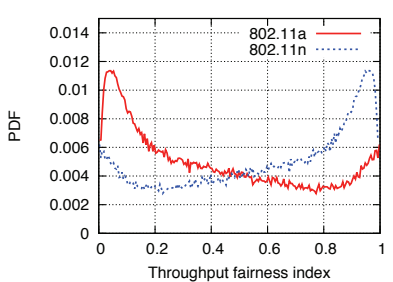

(a) Throughput $\left(F_{s}^{p, c}\right)$

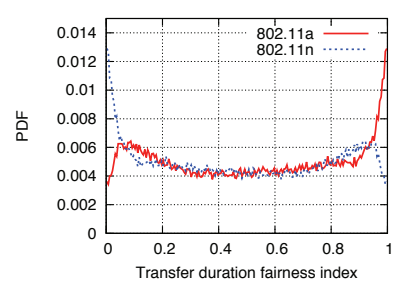

(b) Transfer duration $\left(F_{d}^{p, c}\right)$
Figure 2: PDF of fairness indexes by protocols $(c=36)$

and transfer duration is estimated from the transmission rate and frame length although control frames such as ACK were not captured. From Figure 2(a), the PDFs of the throughput fairness index are biased by protocols, and the index of $802.11 \mathrm{n}$ distributes to larger values; i.e., $\int_{0}^{0.5} y d x \ll \int_{0.5}^{1} y d x$, where $x$ is the throughput fairness index of $802.11 \mathrm{n}$ and $y$ is the PDF of $x$. This means $802.11 n$ has an advantage in throughput. On the other hand, from Figure 2(b), the PDFs of the transfer duration fairness index are flat and similar to each other; i.e., $\int_{0}^{0.5} y d x \simeq \int_{0.5}^{1} y d x$, where $x$ is the transfer duration fairness index of $802.11 \mathrm{n}$ and $y$ is the PDF of $x$. Note that the PDFs have large jumps around at 0 and 1 . We can ignore these jumps because they come from errors in transfer duration estimation due to different ACK mechanisms between 802.11a/g and 802.11n. Hence, the transfer duration is approximately fair between 802.11a and 802.11n. This finding enables us to fairly compare $802.11 \mathrm{a} / \mathrm{g}$ and $802.11 \mathrm{n}$ in coexisting these network.

\section{CONCLUSION}

We had measured and analyzed traffic from hosts using 802.11 protocols in coexisting $802.11 \mathrm{a} / \mathrm{g}$ and $802.11 \mathrm{n}$ wireless networks. We showed that the entropy of PIT of $802.11 \mathrm{n}$ was different from that of $802.11 \mathrm{a} / \mathrm{g}$. However, the entropy-based link type estimation algorithm has difficulty in distinguishing $802.11 n$ hosts from wired hosts. We also showed 802.11a/g and 802.11n were fair in terms of transfer duration though $802.11 n$ gained in terms of throughput.

We will investigate further characteristics of wireless network traffic to identify link types of remote hosts and to find criteria for system design as well as cross-layer characteristics (i.e., TCP).

\section{REFERENÇES}

Pagiannaki, and G. Iannaccone. Detecting 802.11 wireless hosts from remote passive observations. In NETWORKING 200\%, pages 356-367.

[2] W. Wei, B. Wang, C. Zhang, J. Kurose, and D. Towsley. Classification of access network types: Ethernet, wireless LAN, ADSL, cable modem or dialup? Comput. Netw., 52(17):3205-3217, 2008. 